\title{
Correlation of serum anti-Müllerian hormone levels with positive in vitro fertilization outcome using a short agonist protocol
}

\author{
Spyridon D. Mantzavinos, ${ }^{1}$ Nikolaos P. Vlahos, ${ }^{1}$ Demetrios Rizos, ${ }^{2}$ \\ Demetrios Botsis, ${ }^{1}$ Theodoros N. Sergentanis, ${ }^{3}$ \\ Efthimios Deligeoroglou, ${ }^{1}$ Themistoklis Mantzavinos ${ }^{1}$
}

${ }^{1} 2^{\text {nd }}$ Department of Obstetrics and Gynecology, ${ }^{2} 2^{\text {nd }}$ Department of Obstetrics and Gynecology, Hormonologic Laboratory, "Aretaieion" University Hospital, ${ }^{3}$ Department of Hygiene, Epidemiology and Medical Statistics, Medical School; National and Kapodistrian University of Athens, Athens, Greece

\begin{abstract}
OBJECTIVE: We examined the predictive ability of anti-Müllerian hormone (AMH) for clinical pregnancy in women who underwent in vitro fertilization (IVF) cycles in a short agonist protocol. DESIGN: This is a retrospective cohort study of 222 women undergoing their first IVF attempt between June 2010 and March 2016. Multivariate logistic regression analysis was performed to evaluate the independent associations between clinical pregnancy and its possible predictors. RESULTS: $14.9 \%$ of cycles were cancelled, $>3$ oocytes were retrieved in $55.4 \%$ of cycles and embryo transfer was performed in $\mathbf{7 0 . 7 \%}$ of cases. Live birth was the final outcome in $\mathbf{1 9 . 8 \%}$ of subjects, miscarriage occurred in $4.1 \%$, whereas no pregnancy occurred in the remaining 76.1\% of the study sample. The number of oocytes, number of embryos, embryo transfer rate and pregnancy rates were positively associated with serum AMH concentrations $(p<0.001$, for each association). When analyzed by age quartiles, the overall association between AMH and clinical pregnancy rates was evident across all age strata. CONCLUSIONS: Serum AMH levels are a strong predictive marker of clinical pregnancy in women undergoing a short agonist IVF protocol. There is also a strong association with cancellation rate, number of oocytes retrieved, poor response ( $\leq 3$ oocytes), number of embryos, embryo transfer rate and live birth rates.
\end{abstract}

Key words: Anti-Mullerian hormone, Greek population, IVF, Pregnancy rate, Short agonist protocol

Address for correspondence:

Spyridon D. Mantzavinos, Obstetrician-Gynecologist, $2^{\text {nd }}$ Department of Obstetrics and Gynecology, “Aretaieion” University Hospital, National and Kapodistrian University of Athens, Athens, Greece; 16 Aitolias Str., 15341, Agia Paraskevi, Athens, Greece;

Tel.: +30 6945 414487, Fax: +30 2106926124 , E-mail: mantzavinos@yahoo.com

Received: 24-04-2017, Accepted: 04-05-2017 


\section{INTRODUCTION}

Over the past decade there have been hundreds of publications regarding the ability of anti-Müllerian hormone (AMH) to predict a positive pregnancy test in an in vitro fertilization (IVF) programme. Though the results have so far been controversial, it can be considered as established knowledge that serum AMH levels are positively associated with the total number of retrieved oocytes, ${ }^{1-7}$ so that AMH would seem in fact to be highly predictive of poor ovarian response. ${ }^{7-10}$ The association of AMH with ovarian stimulation is even stronger if it is evaluated according to the woman's age. ${ }^{2-4,7}$ If the higher number of oocytes results in a higher number of available good quality embryos, it would be reasonable to expect a higher rate of positive pregnancy tests after in vitro fertilization treatment. ${ }^{11}$

Indeed, a large number of studies have reported a positive correlation between AMH and pregnancy rates. ${ }^{12-20}$ However, there are also a number of studies showing limited predictive ability or no correlation between AMH levels and IVF outcome., 4,7,21-25

The importance of such predictive ability is greater in women of advanced reproductive age due to the reduced success rates of this population.

A meta-analysis by Broer et $\mathrm{a}^{26}$ demonstrated that among various ovarian reserve tests (FSH, AMH, AFC, age), AMH and AFC had similar predictive ability for poor response, but only age had a limited capacity of predicting ongoing pregnancy after IVF. Iliodromiti et $\mathrm{al}^{27}$ in their meta-analysis showed that $\mathrm{AMH}$, independently of age, is correlated with live birth after assisted conception, but with poor predictive accuracy.Tal et $\mathrm{al}^{28}$ published a meta-analysis which concluded that AMH is weakly correlated with clinical pregnancy rates. Finally, one month later Yao et $\mathrm{al}^{29}$ published their meta-analysis which reported that $\mathrm{AMH}$ can predict pregnancy rates after IVF as an independent parameter. It is obvious that after years of publications worldwide, the question of whether AMH predicts pregnancy after IVF still remains unresolved.

In the present study we investigate the correlation between serum AMH levels and pregnancy rate in a population of women undergoing their first IVF cycle using a short agonist protocol.

\section{MATERIALS AND METHODS}

We studied retrospectively the IVF outcomes of patients undergoing their first IVF cycle, between June 2010 and March 2016.

Inclusion criteria for the study were:

1) The patient underwent her first IVF cycle,

2) Serum AMH measurement within 3 months before the beginning of the IVF cycle,

3) No presence of a prolactin or thyroid disorder, or a well treated disorder under continuous follow-up,

4) Ovarian stimulation was carried out using a short agonist protocol and all embryo transfers were carried out on a fresh cycle.

Exclusion criteria:

1) Serum AMH levels unknown or measured more than 3 months before the IVF cycle,

2) Donor egg cycles,

3) Natural cycles,

4) Frozen-thawed cycles.

In couples with unexplained infertility, the duration of infertility was at least 1 year before IVF for women $<35$ years and at least 6 months for women $\geq 35$ years of age. In cases of tubal factor, as well as of male factor, the decision to proceed with an IVF/ ICSI attempt was made as soon as the couple presented with the diagnosis of the cause of infertility. Normal weight status was defined as body mass index (BMI) $18.5-24.9 \mathrm{~kg} / \mathrm{m}^{2}$, overweight as $25-29.9 \mathrm{~kg} / \mathrm{m}^{2}$, obesity as BMI $\geq 30 \mathrm{~kg} / \mathrm{m}^{2}$ and underweight as BMI $<18.5 \mathrm{~kg} / \mathrm{m}^{2}$.

\section{COH PROTOCOL}

We used a short GnRH agonist protocol (flare-up protocol).

The stimulation began with a GnRH agonist, either leuprorelin acetate (Daronda $14 \mathrm{mg} / 2.8 \mathrm{ml}$ ) or triptorelin (Arvekap $0.1 \mathrm{mg}$ ) on Day 2 of the cycle. The daily dose was 20iu of leuprorelin or $0.1 \mathrm{mg}$ of triptorelin, subcutaneously. GnRH agonist was continued until the day of hCG administration. 
The gonadotropin administered was Menopur (Ferring Hellas), Gonal-F (Merck-Serono Europe), Puregon (Merck Sharp \& Dohme Limited-MSD), Merional (Faran) or Altermon (Faran Laboratories). Initial dose was decided based on the woman's age, BMI, antral follicle count (AFC), baseline FSH levels and previous ovarian or other abdominal surgery. Administration began on Day 3 of the cycle and continued until the day of hCG triggering. Ovarian response was monitored on Day 6 using transvaginal ultrasound and serum estradiol levels (E2). Dose was adjusted based on the results and follow-up with ultrasound and E2 was repeated on Day 8 and Day 10. Minimum gonadotropin dose was $150 \mathrm{iu}$ and maximum dose was $450 \mathrm{iu}$.

Urinary hCG (Pregnyl) 5.000-10.000iu (Merck Sharp \& Dohme Limited-MSD) or recombinant hCG (Ovitrelle) 250mg (Merck-Serono Europe Limited) was administered when 2-3 follicles (or one follicle if only one was present) reached a diameter of $16-18 \mathrm{~mm}$.

The procedure was cancelled when no growing follicles were found during the ultrasound follow-up or in cases of poor response with very low levels of serum E2 $(\leq 50 \mathrm{pg} / \mathrm{ml})$.

34 hours later we retrieved the oocytes. We performed rapid oocyte retrieval using a single lumen needle, under sedation. No flushing was used in any case.

Oocyte fertilization was attempted either conventionally or via intracytoplasmic sperm injection (ICSI).

Prior to embryo transfer, embryo quality was assessed using the embryo morphology score. Embryo transfer took place on a fresh cycle, using either a Frydman, Cook or Wallace catheter, under abdominal ultrasound guidance. Transfer was carried out at the cleavage stage (Day 2 or 3) or the blastocyst stage (Day 5 ). Decision was based on the number and quality of available embryos. We transferred only good quality embryos (at least grade 3). In accordance with Greek law, we transferred up to 2 embryos when the woman was less than 40 years of age and up to 4 embryos if the woman was more than 40 years of age. Any further good quality embryos were frozen and were subsequently transferred to a fresh-thawed cycle. However, frozen-thawed cycles were not included in our study.
We supported the luteal phase by using per os or vaginal micronized progesterone (Utrogestan) $200 \mathrm{mg}$ (Angelini Pharma Hellas) three times daily or vaginal progesterone gel $8 \%$ (Vasclor) (Verisfield UK) twice daily until the day of the pregnancy test. If positive, progesterone was continued until 8 weeks of gestation.

We did not prescribe any other medication (such as antibiotics, cortisone, heparin, aspirin).

Measurement of $\beta$-hCG was carried out 2 weeks later and was repeated - if positive - until $\beta \mathrm{HCG}$ levels were $\geq 2000 \mathrm{iu} / \mathrm{l}$. We then conducted a vaginal ultrasound and repeated the examination weekly until a gestational sac was found. We considered clinical pregnancy the presence of a gestational sac with heartbeat, which included miscarriage and live birth as possible outcomes.

\section{HORMONAL MEASUREMENTS}

AMH levels were measured on any day of the woman's menstrual cycle, within 3 months of the beginning of the IVF cycle. AMH concentration was measured using the AMH Gen II Elisa (Beckman Coulter) assay.

FSH levels were measured on the 2nd or 3rd day of the woman's cycle, within 6 months of the IVF cycle.

\section{OUTCOMES}

Primary outcome was clinical pregnancy rate (gestational sac with positive heartbeat). The scientific question of interest was whether serum AMH is independently associated with clinical pregnancy in women undergoing IVF cycles in a short agonist protocol, taking into account the possible confounding effects of age.

Secondary outcomes were: (1) cancellation rate, (2) number of oocytes retrieved, (3) poor ovarian response ( $\leq 3$ oocytes retrieved), (4) number of embryos, (5) embryotransfer rate, (6) live birth rate; their associations with AMH levels were also examined.

\section{STATISTICAL METHODS}

Regarding descriptive statistics, results for quantitative variables were presented as mean $\pm \mathrm{SD}$; the $95 \%$ 
confidence intervals (CIs) for the mean were also provided. Categorical variables were presented as frequency (percentage); the $95 \%$ CIs for proportions were also provided.

The statistical analysis comprised two steps: univariate and multivariate analysis. Serum AMH was not normally distributed ( $<<0.001$, Shapiro-Wilk test), therefore non-parametric statistical tests were implemented. In the univariate analysis, the associations between serum AMH and the study variables (age, serum FSH, cancellation, number of oocytes, poor response, number of embryos, embryo transfer and clinical pregnancy/live birth) were appropriately evaluated through the Mann-Whitney-Wilcoxon test for independent samples (henceforth designated as MWW for the sake of brevity), or Spearman's rank correlation coefficient.

In the multivariate logistic regression analysis, clinical pregnancy (either live birth or miscarriage) was set as the dependent variable in the model. Age in quartiles and $\mathrm{AMH}$ in quartiles were introduced as independent variables in the model; implementation of quartiles was preferred to the use of continuous variables due to the non-linear pattern of associations implicating the aforementioned covariates (data not shown). The lowest quartiles were set as reference categories for both independent variables. Finally, a stratification of the association between AMH levels and clinical pregnancy rates, across age quartilesstrata, was also undertaken for the clearer presentation of underlying results. The statistical analysis was performed using STATA/SE version 13 statistical software (Stata Corp., College Station, TX, USA).

\section{RESULTS}

Descriptive statistics are summarized in Table 1. The mean age of the study sample $(\mathrm{n}=222)$ was 38.7 years (95\%CI: 38.1-39.3 years, range 27-49 years). AMH values ranged between 0.03 and $11.05 \mathrm{ng} / \mathrm{mL}$ (1.36 $\pm 1.66 \mathrm{ng} / \mathrm{mL}, 95 \% \mathrm{CI}: 1.14-1.58 \mathrm{ng} / \mathrm{mL})$. Regarding BMI, 33 women $(14.9 \%$, 95\%CI:10.5\%-20.2\%) were overweight, 17 (7.7\%, 95\%CI: 4.5\%-12.0\%) were obese and $9(4.0 \%, 95 \% \mathrm{CI}: 1.9 \%-7.6 \%)$ were underweight. 43 women (19.4\%, 95\%CI: 14.4\%$25.2 \%)$ smoked $1-10$ cigarettes per day and 27 (12.2\%, 95\% CI: 8.2\%-17.2\%) smoked $>10$ cigarettes per day.
Table 1. Description of the study sample

\begin{tabular}{lcc}
\hline Continuous variables & Median & Mean $\mathbf{( 9 5 \% C I )}$ \\
\hline Age (years) & 39 & $38.7(38.1-39.3)$ \\
AMH (ng/mL) & 0.7 & $1.36(1.14-1.58)$ \\
FSH (IU/L) & 7.1 & $9.2(8.2-10.2)$ \\
Number of oocytes & 4 & $4.6(4.1-5.2)$ \\
Number of embryos & 2 & $3.0(2.6-3.4)$ \\
\hline
\end{tabular}

Categorical variables $\quad$ Frequencies $\quad$ Proportion \%

$(95 \%$ CI $)$

\begin{tabular}{|c|c|c|}
\hline \multicolumn{3}{|l|}{ BMI classification } \\
\hline Underweight & $9 / 222$ & $4.0(1.9-7.6)$ \\
\hline Normal weight & $163 / 222$ & $73.4(67.1-79.1)$ \\
\hline Overweight & $33 / 222$ & $14.9(10.5-20.2)$ \\
\hline Obese & $17 / 222$ & $7.7(4.5-12.0)$ \\
\hline \multicolumn{3}{|l|}{ Smoking } \\
\hline No & $152 / 222$ & $68.5(61.9-74.5)$ \\
\hline $1-10$ cigarettes per day & $43 / 222$ & $19.4(14.4-25.2)$ \\
\hline$>10$ cigarettes per day & $27 / 222$ & $12.2(8.2-17.2)$ \\
\hline \multicolumn{3}{|l|}{ Cancellation } \\
\hline No & $189 / 222$ & $85.1(79.8-89.5)$ \\
\hline Yes & $33 / 222$ & $14.9(10.5-20.2)$ \\
\hline \multicolumn{3}{|l|}{ Poor response } \\
\hline Cancellation or 0 & $37 / 222$ & $16.7(12.0-22.2)$ \\
\hline 1-3 oocytes & $62 / 222$ & $27.9(22.1-34.3)$ \\
\hline$>3$ oocytes & $123 / 222$ & $55.4(48.6-62.1)$ \\
\hline \multicolumn{3}{|l|}{ Embryo transfer } \\
\hline Cancellation or zero embryos & $65 / 222$ & $29.3(23.4-35.7)$ \\
\hline Embryo transfer & $157 / 222$ & $70.7(64.3-76.6)$ \\
\hline \multicolumn{3}{|l|}{ Pregnancy/live birth } \\
\hline No pregnancy & $169 / 222$ & $76.1(70.0-81.6)$ \\
\hline Miscarriage & $9 / 222$ & $4.1(1.9-7.6)$ \\
\hline Live birth & $44 / 222$ & $19.8(14.8-25.7)$ \\
\hline
\end{tabular}

The procedure was cancelled in $14.9 \%(95 \% \mathrm{CI}$ : $10.5 \%-20.2 \%$ ) of cases, $>3$ oocytes were retrieved in $55.4 \%$ (95\% CI: $48.6 \%-62.1 \%$ ) of procedures and embryo transfer was performed in $70.7 \%$ (95\%CI: $64.3 \%-76.6 \%$ ) of cases. Live birth was the final outcome in $19.8 \%$ (95\% CI: $14.8 \%-25.7 \%)$ of subjects, miscarriage occurred in 4.1\% (95\% CI: 1.9\%-7.6\%), whereas in the remaining $76.1 \%$ of the study sample (95\% CI: 70.0\%-81.6\%) no pregnancy was achieved.

The associations of study variables with serum 
Table 2. Association of study variables with serum AMH levels

\begin{tabular}{|c|c|c|c|}
\hline & N (\%) & $\mathrm{AMH}(\operatorname{mean} \pm \mathrm{SD})$ & $\mathbf{p}$ \\
\hline \multicolumn{4}{|l|}{$\overline{\text { Age }}$} \\
\hline Quartile $1(27-35)$ & $52(23.4)$ & $2.37 \pm 2.30$ & $<0.001^{\mathrm{s}}$ \\
\hline Quartile $2(36-38)$ & $47(21.2)$ & $1.28 \pm 1.68$ & \\
\hline Quartile 3 (39-41) & $59(26.6)$ & $1.11 \pm 1.19$ & \\
\hline Quartile 4 (42-49) & $64(28.8)$ & $0.82 \pm 0.86$ & \\
\hline FSH (IU/L) & & & $<0.001^{\mathrm{S}}$ \\
\hline Quartile 1 (0.4-5.1) & $53(23.9)$ & $2.31 \pm 2.30$ & \\
\hline Quartile $2(5.2-7.0)$ & $58(26.1)$ & $1.76 \pm 1.66$ & \\
\hline Quartile 3 (7.1-10.1) & $55(24.8)$ & $0.90 \pm 0.76$ & \\
\hline Quartile 4 (10.2-64.8) & $56(25.2)$ & $0.49 \pm 0.74$ & \\
\hline Cancellation & & & $<0.001^{\mathrm{MWW}}$ \\
\hline No & $189(85.1)$ & $1.54 \pm 1.72$ & \\
\hline Yes & $33(14.9)$ & $0.30 \pm 0.33$ & \\
\hline Number of oocytes & & & $<0.001^{\mathrm{s}}$ \\
\hline Quartile $1(0-1)$ & $54(24.3)$ & $0.30 \pm 0.28$ & \\
\hline Quartile $2(2-3)$ & $45(20.3)$ & $0.82 \pm 0.73$ & \\
\hline Quartile 3 (4-6) & $62(27.9)$ & $1.35 \pm 1.43$ & \\
\hline Quartile 4 (7-28) & $61(27.5)$ & $2.70 \pm 2.11$ & \\
\hline Poor response & & & $<0.001^{\mathrm{S}}$ \\
\hline Cancellation or 0 & $37(16.7)$ & $0.30 \pm 0.32$ & \\
\hline 1-3 oocytes & $62(27.9)$ & $0.67 \pm 0.68$ & \\
\hline$>3$ oocytes & $123(55.4)$ & $2.02 \pm 1.92$ & \\
\hline Number of embryos & & & $<0.001^{\mathrm{s}}$ \\
\hline Quartile $1(0)$ & $49(22.1)$ & $0.32 \pm 0.33$ & \\
\hline Quartile 2 (1) & $27(12.1)$ & $0.66 \pm 0.68$ & \\
\hline Quartile $3(2-4)$ & $89(40.1)$ & $1.26 \pm 1.33$ & \\
\hline Quartile 4 (5-16) & $57(25.7)$ & $2.73 \pm 2.15$ & \\
\hline Embryo transfer & & & $<0.001^{\mathrm{MWW}}$ \\
\hline Cancellation or zero embryos & $65(29.3)$ & $0.56 \pm 1.11$ & \\
\hline Embryo transfer & $157(70.7)$ & $1.69 \pm 1.73$ & \\
\hline Pregnancy/live birth & & & $<0.001^{\mathrm{KW}}$ \\
\hline No pregnancy & $169(76.1)$ & $0.95 \pm 1.14$ & \\
\hline Miscarriage & $9(4.1)$ & $1.40 \pm 1.05$ & \\
\hline Live birth & $44(19.8)$ & $2.92 \pm 2.36$ & \\
\hline
\end{tabular}

MWW: p-value derived from Mann-Whiney-Wilcoxon test for independent samples, S: p-value derived from Spearman's rank correlation coefficient.

AMH levels are presented in Table 2. Women's age correlated inversely with serum AMH (Spearman's rho $=-0.285, \mathrm{p}<0.001)$; serum FSH also correlated in- versely with AMH (Spearman's rho=-0.485, $\mathrm{p}<0.001$ ). Cancelled procedures presented with significantly lower AMH levels $(0.30 \pm 0.33$ vs. $1.54 \pm 1.72 ; \mathrm{p}<0.001$, 
MWW test). The number of oocytes was positively associated with serum AMH concentrations (Spearman's rho $=0.679, p<0.001$ ), as evident also in the response analysis focusing on $>3$ oocytes (Spearman's rho $=0.602, \mathrm{p}<0.001)$. Accordingly, the number of embryos positively correlated with serum AMH concentrations (Spearman's rho $=0.644, \mathrm{p}<0.001$ ). Procedures leading to embryo transfer presented with significantly higher serum AMH levels $(1.69 \pm 1.73$ vs. $0.56 \pm 1.11 \mathrm{ng} / \mathrm{mL} ; \mathrm{p}<0.001$, MWW test). Procedures leading to live birth presented with the highest serum AMH levels $(2.92 \pm 2.36 \mathrm{ng} / \mathrm{mL})$, followed by miscarriage cases $(1.40 \pm 1.05 \mathrm{ng} / \mathrm{mL})$, whereas the lowest AMH levels $(0.95 \pm 1.14 \mathrm{ng} / \mathrm{mL})$ were noted in non-pregnant cases $(\mathrm{p}<0.001$, Kruskal-Wallis test).

The results of the multivariate logistic regression analysis examining the associations between pregnancy, age (in quartiles) and AMH (in quartiles) are presented in Table 3. Compared to the lowest AMH quartile, quartile 2, quartile 3 , quartile 4 were independently associated with 5-fold (adjusted $\mathrm{OR}=5.41,95 \% \mathrm{CI}$ : 1.08-27.06, $\mathrm{p}=0.040$ ), 8-fold (adjusted $\mathrm{OR}=8.45$, 95\%CI: $1.75-40.83, \mathrm{p}=0.008$ ) and 30 -fold (adjusted $\mathrm{OR}=30.43,95 \% \mathrm{CI}: 6.37-145.47, \mathrm{p}<0.001)$ increased odds of pregnancy. On the other hand, regarding age, only the highest quartile (42-49 years) was indepen-

Table 3. Results of the multivariate logistic regression analysis examining the associations between clinical pregnancy, age and AMH quartiles. Bold cells denote statistically significant associations

\begin{tabular}{|c|c|c|c|}
\hline & Clinical Pregnancy rate (\%) & OR $(95 \% \mathrm{CI})$ & $\mathbf{p}$ \\
\hline \multicolumn{4}{|l|}{ Age } \\
\hline Quartile 1 (27-35) & $38.5(20 / 52)$ & Ref. & \\
\hline Quartile 2 (36-38) & $31.9(15 / 47)$ & $1.50(0.57-3.93)$ & 0.408 \\
\hline Quartile 3 (39-41) & $22.0(13 / 59)$ & $0.81(0.32-2.06)$ & 0.662 \\
\hline Quartile 4 (42-49) & $7.8(5 / 64)$ & $0.25(0.08-0.77)$ & 0.016 \\
\hline \multicolumn{4}{|l|}{$\mathrm{AMH}(\mathrm{ng} / \mathrm{mL})$} \\
\hline Quartile $1(0.03-0.29)$ & $3.9(2 / 52)$ & Ref. & \\
\hline Quartile $2(0.30-0.71)$ & $14.5(9 / 62)$ & $5.41(1.08-27.06)$ & 0.040 \\
\hline Quartile 3 (0.72-1.77) & $23.6(13 / 55)$ & $8.45(1.75-40.83)$ & 0.008 \\
\hline Quartile 4 (1.78-11.05) & $54.7(29 / 53)$ & $30.43(6.37-145.47)$ & $<0.001$ \\
\hline
\end{tabular}

Table 4. Subgroup analyses regarding the association of AMH with clinical pregnancy in the age quartiles

\begin{tabular}{|c|c|c|c|}
\hline & $\mathbf{N}(\%)$ & $\mathrm{AMH}(\mathrm{mean} \pm \mathrm{SD}, \mathrm{ng} / \mathrm{mL})$ & $\mathbf{p}$ \\
\hline Age quartile $1(27-35)$ & & & $<0.001^{\mathrm{MWW}}$ \\
\hline Non-pregnant & $32(61.5)$ & $1.46 \pm 1.60$ & \\
\hline Pregnant & $20(38.5)$ & $3.83 \pm 2.53$ & \\
\hline Age quartile $2(36-38)$ & & & $<0.001^{\mathrm{MWw}}$ \\
\hline Non-pregnant & $32(68.1)$ & $0.69 \pm 0.97$ & \\
\hline Pregnant & $15(31.9)$ & $2.55 \pm 2.16$ & \\
\hline Age quartile $3(39-41)$ & & & $0.018^{\mathrm{MWW}}$ \\
\hline Non-pregnant & $46(78.0)$ & $1.02 \pm 1.26$ & \\
\hline Pregnant & $13(22.0)$ & $1.43 \pm 0.84$ & \\
\hline Age quartile 4 (42-49) & & & $0.438^{\mathrm{MWW}}$ \\
\hline Non-pregnant & $59(92.2)$ & $0.76 \pm 0.69$ & \\
\hline Pregnant & $5(7.8)$ & $1.52 \pm 2.05$ & \\
\hline
\end{tabular}


dently associated with decreased odds of pregnancy (adjusted $\mathrm{OR}=0.25,95 \% \mathrm{CI}: 0.08-0.77, \mathrm{p}=0.016$ ), compared to the lowest quartile.

The subgroup analyses by age quartile (Table 4) clearly supported the reproducibility of the overall association between AMH and pregnancy rates across all age strata. Specifically, the AMH difference between pregnant and non-pregnant women reached significance in age quartile 1 ( $<<0.001$, MWW test), quartile 2 ( $p$ $<0.001$, MWW test) and quartile $3(\mathrm{p}=0.018$, MWW test). Despite a sizable numerical difference $(1.52 \pm 2.05$ vs. $0.76 \pm 0.69 \mathrm{ng} / \mathrm{mL}$ ), statistical significance was not reached in age quartile 4 ( $\mathrm{p}=0.438$, MWW test); nevertheless, this seems only to be due to the small number of pregnancy events in this stratum.

\section{DISCUSSION}

$\mathrm{AMH}$ has been established as a quantitative marker of ovarian reserve and is widely used as a predictive marker of quantitative ovarian response in the majority of IVF Centers worldwide. Most studies in the literature have demonstrated that $\mathrm{AMH}$ concentrations are positively associated with oocyte yield after ovarian stimulation. We would expect that a higher number of oocytes would result in a higher pregnancy rate. Indeed, the studies of Van der Gaast et al, ${ }^{30}$ Sunkara et $\mathrm{al},{ }^{31} \mathrm{Mc}$ Avey et $\mathrm{al}^{32}$ and $\mathrm{Ji}$ et $\mathrm{al}^{33}$ showed a positive correlation between number of oocytes and pregnancy rates (up to 15 oocytes). However, the association between AMH levels and IVF success has been controversial. While many studies show AMH to be a good predictive marker of live birth rates, others support the view that $\mathrm{AMH}$ does not have a significant predictive ability. Recent meta-analyses did not in fact confirm this relationship. ${ }^{26,27}$ In this setting, our study demonstrates a strong positive association of AMH levels with pregnancy rates.

Our study sample included a high proportion of women of advanced reproductive age (mean age 38.7 years, $81.6 \%$ of cycles were from women $\geq 35$ years old). Accordingly, we found a low level of mean AMH $(1.36 \mathrm{ng} / \mathrm{ml})$. This resulted in a relatively low percentage of pregnancies $(23.9 \%)$. However, a number of studies have indicated that AMH has a high predictive accuracy in women of advanced reproductive age.,34

Serum AMH levels showed an inverse correlation with age, as did AMH with FSH levels. This result confirms the reliability of our data regarding the population of our study.

We found a significant correlation between serum $\mathrm{AMH}$ levels and cancellation rate, as well as number of oocytes retrieved. AMH levels also predicted women with poor response ( $\leq 3$ oocytes). These findings are seen in most studies reporting the predictive ability of AMH for ovarian responsiveness to stimulation, ${ }^{2-6}$ especially for the prediction of poor responders. ${ }^{1,7-10}$

Moreover, in our study serum AMH levels exhibited significant correlations with number of embryos, embryo transfer rates and pregnancy rates. Our findings substantiate the significant predictive ability of serum AMH for pregnancy rates, as have many recent studies. ${ }^{12-20}$

The predictive ability of AMH for pregnancy rates remained significant when we stratified serum AMH in quartiles. Compared with the first quartile of AMH levels, we found a 5-fold increase in odds of pregnancy in the second quartile, an 8-fold in the third quartile and a 30 -fold in the fourth quartile. Moreover, when stratifying age into quartiles we found a statistically significant correlation of AMH with pregnancy rates in all age groups except in women aged $42-49$ years where the difference of AMH levels was obvious but did not reach significance due to the very small number of pregnancies occurring in this group. The prognostic value of AMH in our study was strong in all age groups, while other studies in the literature show that AMH's predictive ability depends on the woman's age. .,3,18 $^{2}$

In our research, the cut-off point of AMH under which no pregnancies were noted was $0.27 \mathrm{ng} / \mathrm{ml}$. According to several other studies, ${ }^{20}$ the very low AMH cut-off level does not allow a clinical interpretation of very low AMH levels, which would justify denial of assisted reproduction techniques to an infertile couple.

In our clinic, we predominantly use the short agonist protocol for ovarian stimulation, especially in women of advanced reproductive age ( $\geq 38$ years old). Given the study design, we could not evaluate if patients might have better results using another protocol.

One limitation of our study was the fact that the antral follicle count (AFC) was not available in the 
total dataset, a known prognostic factor for pregnancy after an IVF cycle. ${ }^{8,9,35}$ Another limitation is the retrospective nature of our study, including the different types of medication used (gonadotropins, GnRH agonists, hCG, luteal phase support), the different duration and total dose of gonadotropins required, as well as the embryo transfer of either Day 2/Day 3 embryos or blastocysts. Finally, the sample size of the study was relatively limited ( $n=222)$, even if our results reached statistical significance.

Duration of stimulation was merely a proxy index of the duration of each woman's cycle and therefore was not deemed meaningful in the context of the scientific analysis. In turn, the total dose of the gonadotropins was in part determined by the differences in the duration of stimulation and, accordingly, it was not included in the analysis. Moreover, in some cases of poor response (fewer than 3 growing follicles), the dose of gonadotropins was reduced during follow-up in order to reduce the cost of the attempt. However, this would be a confounding factor if total gonadotropin dose was included in the study, since it would seem that a woman with a low AMH and a subsequent poor response would actually need fewer gonadotropins for ovarian stimulation. Also, we did not aim to examine the total dose of gonadotropins, since we used different types of gonadotropins (recombinant/human menopausal/highly purified).

Some other parameters such as the number of follicles $>11 \mathrm{~mm}$ as well as E2 levels on the day of triggering were taken into account when deciding to proceed with the hCG injection, but these parameters were not retained for the purpose of this study.

Regarding the validity of our results, it should be underlined that our study was designed based on the first IVF attempts. If we had included subsequent IVF attempts, then other parameters would have been relevant, such as the determination of the dose based on previous success or failure, use of a different fertilization procedure (IVF/ICSI), possibly higher doses in previously poor responders, as well as the selection bias (women undergoing additional cycles may differ from those entering only one attempt in several aspects, such as age, economic status and previous response).

Our research concludes that serum AMH levels are a valuable tool in the procedure of counselling couples regarding their possibilities of a favorable outcome before entering an IVF programme. It also facilitates our decisions when we are facing repeated failures of an IVF outcome. However, since we observe pregnancies even in women with very low AMH levels, we cannot refuse an IVF procedure to a woman based solely on her AMH values.

To our knowledge, this is one of the few studies regarding the predictive ability of AMH in a Greek population of IVF patients. Moreover, most of the published studies in the literature have investigated the correlation of AMH with IVF success in a longprotocol or an antagonist protocol. In December 2016, Kotanidis et al published a prospective study of 120 women undergoing a short agonist IVF protocol in their first IVF treatment. They found a strong positive correlation between AMH and number of oocytes, as well as a positive association with the availability of supernumerary embryos suitable for cryopreservation. However, they did not investigate the possible association of AMH levels with pregnancy rate. ${ }^{36}$

Our study used a short-agonist stimulation protocol, which would be indicated in a population of expected low responders. Counselling these women often proves to be very difficult, since live birth rates are relatively low in this patient group.

\section{ETHICAL CONSIDERATIONS}

This research was approved by the Research Committee of Aretaieion University Hospital number S-111/23-02-2010.

\section{FUNDING}

There was no outside funding provided for this research.

\section{CONFLICT OF INTEREST}

The authors do not have any conflict of interest relevant to this article.

\section{STATEMENT}

This manuscript has not been published or submitted elsewhere. 


\section{GRANTS OR FELLOWSHIP SUPPORTS}

There was no grant or fellowship support provided for this research.

\section{REFERENCES}

1. Al-Azemi M, Killick SR, Duffy S, et al, 2011 Multimarker assessment of ovarian reserve predicts oocyte yield after ovulation induction. Hum Reprod 26: 414422.

2. Barad DH, Weghofer A, Gleicher N, 2011 Utility of age-specific serum anti-Müllerian hormone concentrations. Reprod Biomed Online 22: 284-291.

3. Honnma H, Baba T, Sasaki M, et al, 2012 Different ovarian response by age in an anti-Müllerian hormonematched group undergoing in vitro fertilization. J Assist Reprod Genet 29: 117-125.

4. Ferté-Delbende C, Catteau-Jonard S, Barrière P, Dewailly D, 2010 Evaluation of the ovarian reserve. J Gynecol Obstet Biol Reprod (Paris) 39: Suppl 2: 27-33.

5. van Rooij IA, Broekmans FJ, te Velde ER, et al, 2002 Serum anti-Müllerian hormone levels: a novel measure of ovarian reserve. Hum Reprod 17: 3065-3071.

6. Fréour T, Mirallié S, Colombel A, Bach-Ngohou K, Masson D, Barrière P, 2006 Anti-Müllerian hormone: clinical relevance in assisted reproductive therapy. Ann Endocrinol (Paris) 67: 567-574.

7. McIlveen M, Skull JD, Ledger WL, 2007 Evaluation of the utility of multiple endocrine and ultrasound measures of ovarian reserve in the prediction of cycle cancellation in a high-risk IVF population. Hum Reprod 22: 778-785.

8. Tolikas A, Tsakos E, Gerou S, Prapas Y, Loufopoulos A, 2011 Anti-Müllerian hormone (AMH) levels in serum and follicular fluid as predictors of ovarian response in stimulated (IVF and ICSI) cycles. Hum Fertil (Camb) 14: 246-253.

9. Nardo LG, Gelbaya TA, Wilkinson H, et al, 2009 Circulating basal anti-Müllerian hormone levels as predictor of ovarian response in women undergoing ovarian stimulation for in vitro fertilization. Fertil Steril 92: 1586-1593.

10. Andersen AN, Witjes H, Gordon K, Mannaerts B, 2011 Predictive factors of ovarian response and clinical outcome after IVF/ICSI following a $\mathrm{rFSH} / \mathrm{GnRH}$ antagonist protocol with or without oral contraceptive pre-treatment. Hum Reprod 26: 3413-3423.

11. Sills ES, Collins GS, Brady AC, et al, 2011 Bivariate analysis of basal serum anti-Müllerian hormone measurements and human blastocyst development after IVF. Reprod Biol Endocrinol 9: 153.

12. Brodin T, Hadziosmanovic N, Berglund L, Olovsson M, Holte J, 2013 Anti-Müllerian hormone levels are strongly associated with live-birth rates after assisted reproduction. J Clin Endocrinol Metab 98: 1107-1114.

13. Arce JC, La Marca A, Mirner Klein B, Nyboe Andersen A, Fleming R, 2013 Anti-Müllerian hormone in gonadotropin releasing-hormone antagonist cycles: prediction of ovarian response and cumulative treatment outcome in good-prognosis patients. Fertil Steril 99: 1644-1653.

14. Nelson SM, Yates RW, Lyall H, et al, 2009 Anti-Müllerian hormone-based approach to controlled ovarian stimulation for assisted conception. Hum Reprod 24: 867-875.

15. Honnma H, Baba T, Sasaki M, et al, 2013 Serum antiMüllerian hormone levels affect the rate of ongoing pregnancy after in vitro fertilization. Reprod Sci 20: 51-59.

16. Kini S, Li HW, Morrell D, Pickering S, Thong KJ, 2010 Anti-Müllerian hormone and cumulative pregnancy outcome in in-vitro fertilization. J Assist Reprod Genet 27: 449-456.

17. La Marca A, Sighinolfi G, Radi G, et al, 2010 AntiMüllerian hormone (AMH) as a predictive marker in assisted reproductive technology (ART). Hum Reprod Update 16: 113-130.

18. Wang JG, Douglas NC, Nakhuda GS, et al, 2010 The association between anti-Müllerian hormone and IVF pregnancy outcomes is influenced by age. Reprod Biomed Online 21: 757-761.

19. Eldar-Geva T, Ben-Chetrit A, Spitz IM, et al, 2005 Dynamic assays of inhibin B, anti-Müllerian hormone and estradiol following FSH stimulation and ovarian ultrasonography as predictors of IVF outcome. Hum Reprod 20: 3178-3183.

20. Gleicher N, Weghofer A, Barad DH, 2010 Anti-Müllerian hormone $(\mathrm{AMH})$ defines, independent of age, low versus good live-birth chances in women with severely diminished ovarian reserve. Fertil Steril 94: 2824-2827.

21. Lie Fong S, Baart EB, Martini E, et al 2008 AntiMüllerian hormone: a marker for oocyte quantity, oocyte quality and embryo quality? Reprod Biomed Online 16: 664-670.

22. Guerif F, Lemseffer M, Couet ML, Gervereau O, Ract V, Royere D, 2009 Serum antimullerian hormone is not predictive of oocyte quality in in vitro fertilization. Ann Endocrinol (Paris) 70: 230-234.

23. Riggs R, Kimble T, Oehninger S, et al, 2011 AntiMüllerian hormone serum levels predict response to controlled ovarian hyperstimulation but not embryo quality or pregnancy outcome in oocyte donation. Fertil Steril 95: 410-412.

24. Bhide P, Gudi A, Shah A, Timms P, Grayson K, Homburg R, 2013 Anti-Müllerian hormone as a predictor of pregnancy following IVF. Reprod Biomed Online 26: 247-252.

25. Anckaert E, Smitz J, Schiettecatte J, Klein BM, Arce JC, 2012 The value of anti-Müllerian hormone measurement 
in the long GnRH agonist protocol: association with ovarian response and gonadotrophin-dose adjustments. Hum Reprod 27: 1829-1839.

26. Broer SL, van Disseldorp J, Broeze KA, et al, 2013 Added value of ovarian reserve testing on patient characteristics in the prediction of ovarian response and ongoing pregnancy: an individual patient data approach. Hum Reprod Update 19: 26-36.

27. Iliodromiti S, Kelsey TW, Wu O, Anderson RA, Nelson SM, 2014 The predictive accuracy of anti-Müllerian hormone for live birth after assisted conception: a systematic review and meta-analysis of the literature. Hum Reprod Update 20: 560-570.

28. Tal R, Tal O, Seifer BJ, Seifer DB, 2015 Anti-Müllerian hormone as predictor of implantation and clinical pregnancy after assisted conception: a systematic review and meta-analysis. Fertil Steril 103: 119-130.

29. Yao L, Zhang W, Li H, Lin W, 2015 The role of serum $\mathrm{AMH}$ and FF AMH in predicting pregnancy outcome in the fresh cycle of IVF/ICSI: a meta-analysis. Int J Clin Exp Med 8: 1755-1767.

30. van der Gaast MH, Eijkemans MJ, van der Net JB, et al, 2006 Optimum number of oocytes for a successful first IVF treatment cycle. Reprod Biomed Online 13: 476-480.

31. Sunkara SK, Rittenberg V, Raine-Fenning N, Bhattacharya S, Zamora J, Coomarasamy A, 2011 Associa- tion between the number of eggs and live birth in IVF treatment: an analysis of 400135 treatment cycles. Hum Reprod 26: 1768-1774.

32. McAvey B, Zapantis A, Jindal SK, Lieman HJ, Polotsky AJ, 2011, How many eggs are needed to produce an assisted reproductive technology baby: is more always better? Fertil Steril 96: 332-335.

33. Ji J, Liu Y, Tong XH, Luo L, Ma J, Chen Z, 2013, The optimum number of oocytes in IVF treatment: an analysis of 2455 cycles in China. Hum Reprod 28: 2728-2734.

34. Park HJ, Lyu SW, Seok HH, Yoon TK, Lee WS, 2015 Anti-Müllerian hormone levels as a predictor of clinical pregnancy in in vitro fertilization/intracytoplasmic sperm injection-embryo transfer cycles in patients over 40 years of age. Clin Exp Reprod Med 42: 143-148.

35. He YX, Xia R, Chen X, et al, 2013 Estimation of ovarian response using multiple predictors of ovarian reserve in women undergoing in vitro fertilizationembryo transfer. Nan Fang Yi Ke Da Xue Xue Bao 33: $216-220$.

36. Kotanidis L, Nikolettos K, Petousis S, et al, 2016 The use of serum anti-Müllerian hormone (AMH) levels and antral follicle count (AFC) to predict the number of oocytes collected and availability of embryos for cryopreservation in IVF. J Endocrinol Invest 39: 14591464. 